\title{
Hereditary Susceptibility to Breast Cancer: Significance of Age of Onset in Family History and Contribution of BRCA1 and BRCA2
}

\author{
Thomas S. Frank, Amie M. Deffenbaugh, \\ Mark Hulick and Kathryn Gumpper \\ Myriad Genetic Laboratories, Salt Lake City, \\ UT, USA
}

\begin{abstract}
OBJECTIVE: To correlate mutations in BRCA1 and BRCA2 with family history of breast cancer in a first-degree relative for women diagnosed with breast cancer before age 45 who do not have a personal or family history of ovarian cancer.

METHODS: Family history for women with breast cancer diagnosed before age 45 was provided by ordering physicians via a test requisition form designed for this purpose. Gene analysis was performed by dye primer sequencing for the entire coding regions of BRCA1 and BRCA2. Because a personal and family history of ovarian cancer are known to be significantly associated with mutations, women with either were excluded from analysis.

RESULTS: Overall, deleterious mutations in BRCA1 or BRCA2 were identified in 85 of 440 women (19\%) with breast cancer under 45. Mutations were identified in 73 of 276 women $(26 \%)$ with a first degree family history of breast cancer compared to 12 of 164 without $(7 \%)(\mathrm{P}<.0001)$. When results were analyzed by the age of diagnosis in first degree relatives, mutations were identified in 56 of 185 women $(30 \%)$ with at least one first degree relative with breast cancer diagnosed before age 50 compared with 17 of 91 women (19\%), where the first degree family history of breast cancer was at or over age 50 $(\mathrm{P}=.042)$.

CONCLUSION: Among women with breast cancer diagnosed before age 45 , a first-degree relative diagnosed with the disease under age 50 is an indicator of a mutation in BRCAl or BRCA2 even in the absence of a family history of ovarian cancer. Therefore, women diagnosed with early-onset breast cancer should be asked about the age of onset in
\end{abstract}

any first-degree relative diagnosed with the disease, as well as about any family history of ovarian cancer. Mutations in BRCA2 account for a substantial proportion of hereditary breast cancer. Therefore, studies that are limited to BRCA1 or that do not analyze by age of onset of breast cancer in relatives may underestimate the contribution of mutations in BRCAl and BRCA2 to women with early onset breast cancer.

\section{BACKGROUND}

Approximately $7 \%$ of breast cancer arises from inherited genetic mutations [1], the majority of which are in BRCA1 and BRCA2 [24]. Inherited mutations in these genes are associated with an elevated risk of breast cancer of $56 \%$ to $87 \%$ by age $70[5,6]$, and $33 \%$ to $50 \%$ before age $50[6,7]$. In addition, the risk of ovarian cancer by age 70 is $22 \%$ [8] to $44 \%[5,7]$ in carriers of BRCA1 mutations, and $27 \%$ for carriers of mutations in BRCA2 [9].

Although the clinical behavior of breast cancer in BRCA1-BRCA2 mutation carriers appears to be similar to that of non-hereditary breast cancer [10-12], mutations confer an increased risk of a second, contralateral breast cancer $[5,10]$ and a ten-fold increase in the risk of ovarian carcinoma in women who have already been diagnosed with breast cancer [13]. Identifying germline mutations in BRCA1 and BRCA2 therefore has significant implications for the medical management of breast cancer patients as well as their relatives.

It has been shown that a personal or family history of both ovarian and early-onset breast cancer indicates an increased likelihood of an inherited mutation in BRCA1 and BRCA2 [13- 
15]. Many studies of site-specific breast cancer families, however, have analyzed only BRCA1 and may not have evaluated the family history according to the age of diagnosis of breast cancer in relatives. We have analyzed the prevalence of mutations in BRCA1 and BRCA2 in women diagnosed with breast cancer before age 45 without a personal or family history of ovarian cancer We have correlated the results with a history of breast cancer in first-degree relatives (i.e., sister and/or mother) in order to identify women with early-onset breast cancer who should be considered for hereditary breast cancer risk assessment.

\section{OBJECTIVE}

To identify which women with breast cancer before age 45 are most likely to carry mutations in BRCA1 and BRCA2.

\section{MATERIALS AND METHODS}

Sequence analysis of BRCA1 and BRCA2 was provided as a clinical laboratory service (Myriad Genetic Laboratories, Salt Lake City, UT, USA) to women whose personal or family history indicated the possibility of an inherited mutation in BRCA1 or BRCA2. Exons 2-24 of BRCA1 and exons $2-27$ of BRCA2 were amplified using 82 pairs of PCR primers designed to avoid common polymorphisms that might inhibit equal amplification of both alleles. Dye primer sequencing was performed using fluorescent energy transfer primers (Amersham Life Science Inc, Cleveland, OH, USA), the mutant Taq polymerase F667Y and a thermal stable pyrophosphatase (both from Perkin Elmer, Norwalk, CT, USA). Sequencing reaction products were electrophoresed and detected using a Perkin Elmer Applied Biosystems 377 sequencing apparatus. Analysis of sequence data was performed using software developed by the clinical laboratory. All analyses demonstrating mutations were repeated for verification. For the purposes of this study, individuals with missense mutations of unproven clinical significance were not considered 'positive'.

A personal and family history of cancer and ancestry was provided via a routine laboratory requisition form. Because a personal or family history of ovarian cancer is known to be a significant predictor of a mutation in BRCA1 or BRCA2 [13], women who reported with either were deliberately excluded from this analysis. For the purposes of this study, non-invasive intraductal lesions such as ductal carcinoma-insitu were not included as 'cancer'.

\section{RESULTS}

Overall, 85 of 440 women (19\%) with breast cancer diagnosed before age 45 had deleterious mutations, of which 50 occurred in $\mathrm{BRCAl}$ and 35 in BRCA2.

Deleterious mutations in BRCA1 or BRCA2 were identified in 73 of 276 women (26\%) with

Table 1

Mutations in BRCA1 and BRCA2 in women with breast cancer diagnosed before age 45 (without a personal or family history of ovarian cancer).

\begin{tabular}{cccccc}
\hline $\begin{array}{c}\text { First degree } \\
\text { relative with } \\
\text { breast cancer }\end{array}$ & $\begin{array}{c}\text { First degree } \\
\text { relative with } \\
\text { breast cancer } \\
<50 \text { years }\end{array}$ & $\begin{array}{c}\text { Total number of } \\
\text { women analyzed } \\
\text { for BRCA1 and } \\
\text { B0 years }\end{array}$ & $\begin{array}{c}\text { Number of } \\
\text { women with } \\
\text { mutations in } \\
\text { BRCA1 }\end{array}$ & $\begin{array}{c}\text { Number of } \\
\text { women with } \\
\text { mutations in } \\
\text { BRCA2 }\end{array}$ & $\begin{array}{c}\text { Total number of } \\
\text { women with } \\
\text { mutations in BRCA1 } \\
\text { and BRCA2 }\end{array}$ \\
\hline no & no & 164 & $3(1.8 \%)$ & $9(5.5 \%)$ & $12(7.3 \%)$ \\
no & yes & 91 & $7(7.7 \%)$ & $10(11 \%)$ & $17(19 \%)$ \\
yes & no & 152 & $34(22 \%)$ & $13(9 \%)$ & $47(31 \%)$ \\
yes & yes & 33 & $6(18 \%)$ & $3(9 \%)$ & $9(27 \%)$ \\
& Total & 440 & $50(11 \%)$ & $35(7.9 \%)$ & $85(19 \%)$ \\
\hline
\end{tabular}


breast cancer under 45 and a first degree family history of breast cancer at any age, compared to 12 of 164 women (7\%) with breast cancer under 45 who reported having no first degree relatives with the disease $(\mathrm{P}<.0001)$.

When results were analyzed by the age of diagnosis of the first degree relative, mutations were significantly more prevalent in women whose first degree family history included breast cancer before age 50 (56 of $185,30 \%$ ) than women whose first degree family history was limited to breast cancer diagnosed at or over age 50 (17 of $91,19 \%)(\mathrm{P}=.042)$.

The results of this analysis are summarized in Table 1.

\section{CONCLUSIONS}

Among women with breast cancer diagnosed before age 45 , a first degree relative with breast cancer diagnosed before age 50 is significantly more predictive of a mutation in BRCA1 or BRCA2 than a first degree relative with breast cancer diagnosed at or after age 50 .

Mutations in BRCA2 account for a substantial proportion (41\%) of mutations in women with breast cancer diagnosed before age 45 , indicating that studies of BRCA1 alone likely underestimate the hereditary contribution to early-onset breast cancer.

Women diagnosed with breast cancer before age 45 with a family history of breast cancer before age 50 in a mother or sister should be evaluated for the possibility of a hereditary cancer syndrome even in the absence of a personal or family history of ovarian cancer.

\section{References}

[1] Claus, E.B., Schildkraut, J.M., Thompson, W.D. and Risch, N.J. The genetic attributable risk of breast and ovarian cancer. Cancer 77, (1996) 2318-2324.

[2] Zweemer, R.P., Verheijen, R.H.M., Gille, J.J.P., vanDiest, P.J., Pals, G. and Menko, F.H. Clinical and genetic evaluation of thirty ovarian cancer families. American Journal of Obstetrics \& Gynecology 178, (1998) 85-90.

[3] Takahashi, H., Chiu, H.C., Bandera, C.A., et al. Mutations of the BRCA2 gene in ovarian carcinomas. Cancer Res. 56, (1996) 2738-2741.

[4] Stratton, J.F., Gayther, S.A., Russell, P., et al. Contribution of BRCA1 mutations to ovarian cancer. New England Journal of Medicine 336, (1997) 1125-1130.

[5] Ford, D., Easton, D.F., Bishop, D.T., Narod, S.A., Goldgar, D.E. and the Breast Cancer Linkage Consortium. Risks of cancer in BRCA1mutation carriers. Lancet 343, (1994) 692-695.

[6] Struewing, J.P., Hartge, P., Wacholder, S., et al. The risk of cancer associated with specific mutations of BRCA1 and BRCA2 among Ashkenazi Jews. New England Journal of Medicine 336, (1997) 1401-1408.

[7] Easton, D.F., Ford, D., Bishop, D.T. and the Breast Cancer Linkage Consortium. Breast and ovarian cancer incidence in BRCA1-mutation carriers. Am. J. Hum. Genet. 56, (1995) 265-271.

[8] Whittemore, A.S., Gong, G. and Itnyre, J. Prevalence and contribution of BRCAl mutations in breast cancer and ovarian cancer: results from three U.S. population-based case-control studies of ovarian cancer. Am. J. Hum. Genet. 60, (1997) 496-504.

[9] Ford, D., Easton, D.F., Stratton, M., et al. Genetic heterogeneity and penetrance analysis of the BRCA1 and BRCA2 genes in breast cancer families. Am. J. Hum. Genet. 62, (1998) 676689.

[10] Verhoog, L.C., Brekelmans, C.T.M., Seynaeve, C., et al. Survival and tumour characteristics of breast-cancer patients with germline mutations of BRCA1. Lancet 351, (1998) 316-321.

[11] Robson, M., Gilewski, T., Haas, B., et al. BRCAassociated breast cancer in young women. $J$. Clin. Oncol. 16, (1998) 1642-1649.

[12] Jóhannsson, O.T., Ranstam, J., Borg, A. and Olsson, H. Survival of BRCA1 breast and ovarian cancer patients: a population-based study from southern Sweden. J. Clin. Oncol. 16, (1998) 397-404.

[13] Frank, T.S., Manley, S.A., Olopade, O.I., et al. Sequence analysis of BRCA1 and BRCA2: correlation of mutations with family history and ovarian cancer risk. J. Clin. Oncol. 16, (1998) 2417-2425.

[14] Couch, F.J., DeShano, M.L., Blackwood, M.A., et al. BRCA1 mutations in women attending clinics that evaluate the risk of breast cancer. 
New England Journal of Medicine 336, (1997) 1409-1415.

[15] Malone, K.E., Daling, J.R., Thompson, J.D., O'Brien, C.A., Francisco, L.V. and Ostrander, E.A. BRCA1 mutations and breast cancer in the general population. Analyses in women before age 35 years and in women before age 45 years with first-degree family history. JAMA 279, (1998) 922-929. 


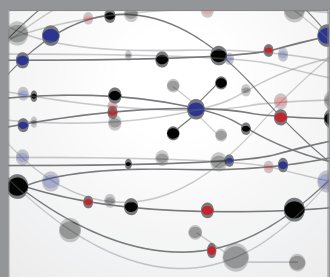

The Scientific World Journal
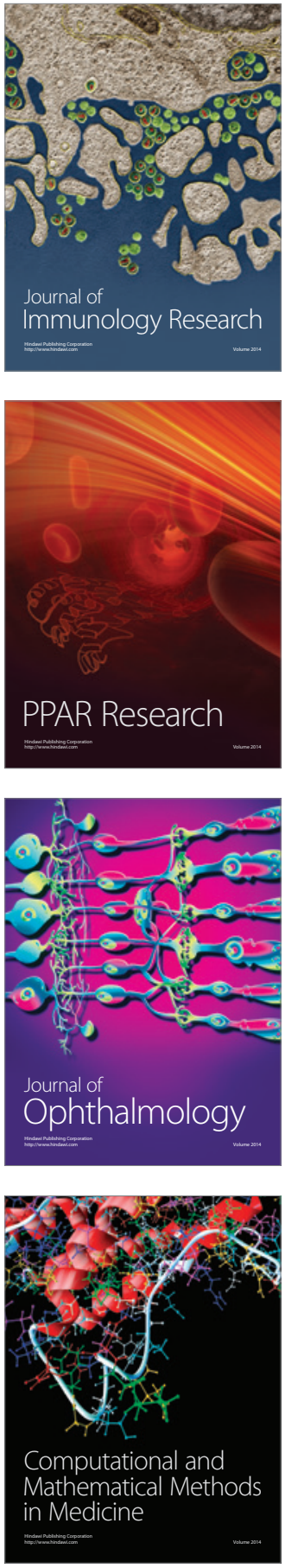

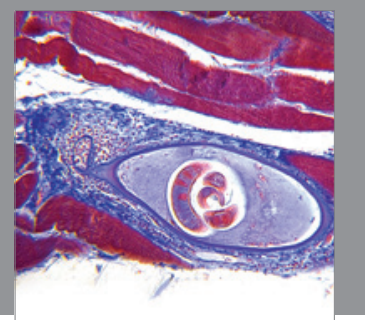

Gastroenterology

Research and Practice
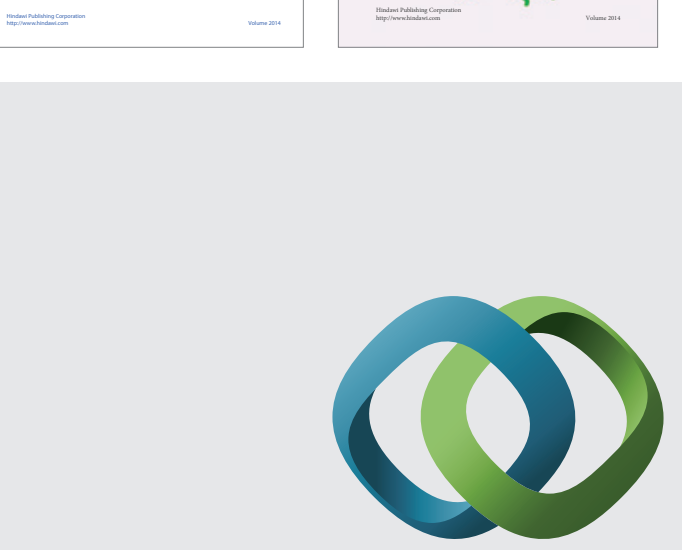

\section{Hindawi}

Submit your manuscripts at

http://www.hindawi.com
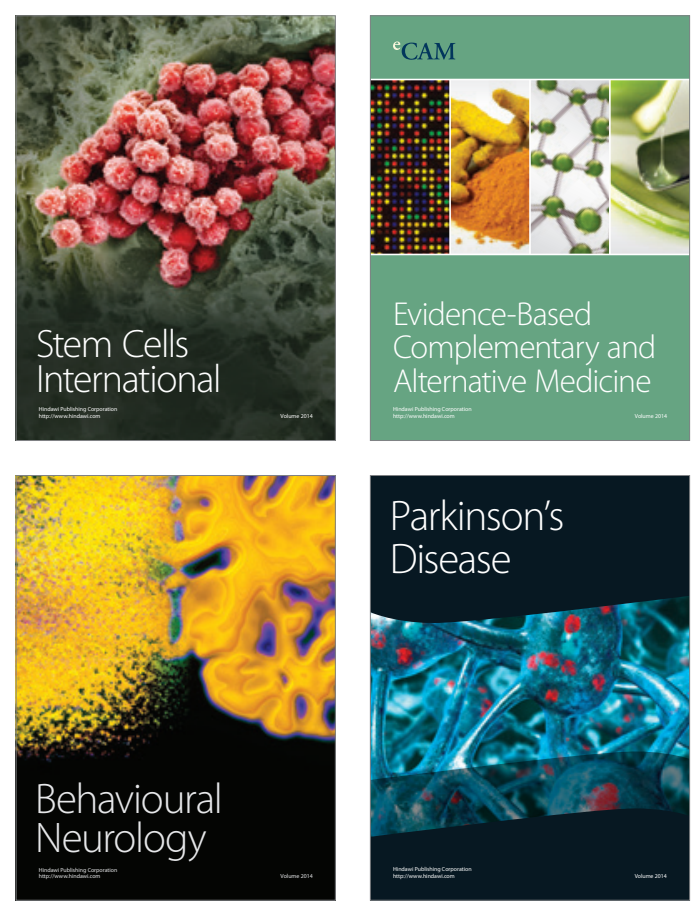

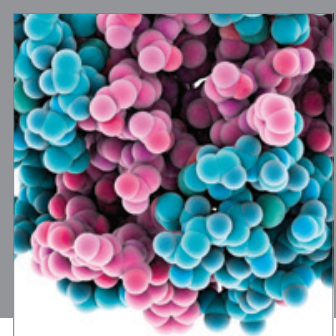

Journal of
Diabetes Research

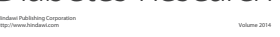

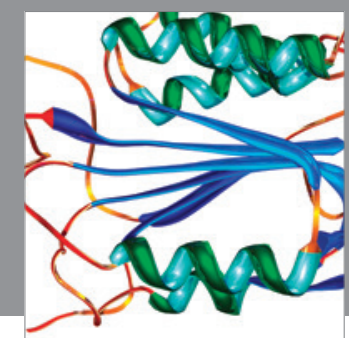

Disease Markers
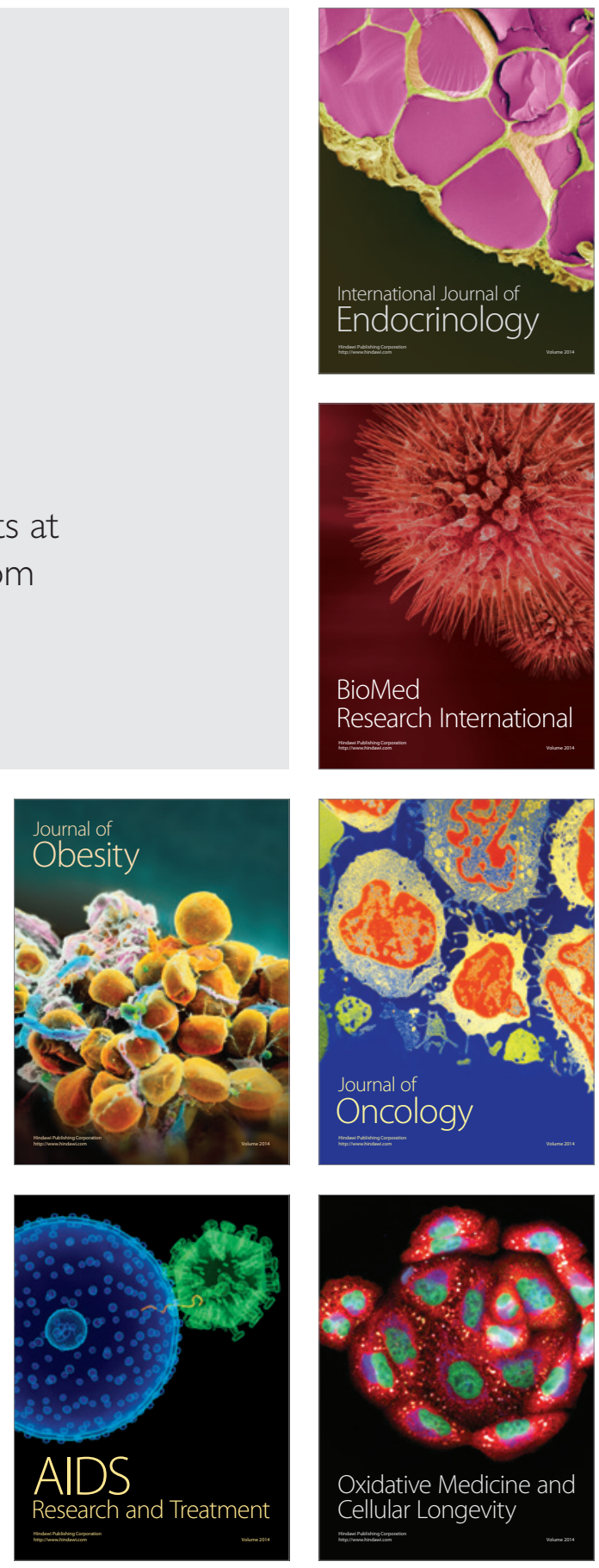